\title{
Social distancing measures differentially affected rats in North America and Tokyo
}

\author{
Yasushi Kiyokawa $^{1}$ (1) $\cdot$ Tsutomu Tanikawa $^{2} \cdot$ Masato Ootaki $^{1} \cdot$ Michael H. Parsons $^{3}$
}

Received: 2 March 2021 / Revised: 19 June 2021 / Accepted: 28 June 2021 / Published online: 6 July 2021

(c) The Author(s), under exclusive licence to Springer-Verlag GmbH Germany, part of Springer Nature 2021

\begin{abstract}
Because rats are commensal organisms that depend on human activities for food, shifts in human behavior will have pronounced effects on local rat populations. In the spring of 2020, social distancing measures were implemented globally to curtail the spread of SARS-CoV-2. This presented a unique opportunity to obtain information regarding the immediate effects of shifts in human behavior on rat populations in a variety of countries. In response to increased sightings of rats in the USA that were reported in American media, we analyzed the changes in the number of public service calls in Tokyo, Japan. We found that the number of calls increased after the implementation of social distancing measures, suggesting that rat sightings had also increased in Tokyo. We then surveyed the changes in the business activities of pest management professionals in the USA, Canada, and Tokyo. We found that the activities were increased in 50 to $60 \%$ of the respondents from the USA and Canada. In contrast, 60 to $70 \%$ of the respondents from Tokyo answered that their activities were not changed. These results implied that, following the implementation of social distancing measures, rat infestations increased in North America, but not in Tokyo. The survey also suggested that roof rats were considered to be the predominant rodent species in Tokyo. This may account for the limited infestations in Tokyo because roof rats are more sedentary than brown rats. Taken together, our findings suggest that social distancing measures differentially affected rat populations in North America and Tokyo.
\end{abstract}

Keywords Brown rats $\cdot$ COVID-19 $\cdot$ Questionnaires $\cdot$ Rat infestation $\cdot$ Roof rats

\section{Key message}

- We assessed the immediate effects of social distancing measures against COVID-19 on rats

- Business activities among pest management professionals increased in North America

- Even if rat sightings increased, changes in business activities were minor in Tokyo

Communicated by Christian Imholt.

Yasushi Kiyokawa

akiyo@mail.ecc.u-tokyo.ac.jp

1 Laboratory of Veterinary Ethology, The University of Tokyo, 1-1-1 Yayoi, Bunkyo-ku, Tokyo 113-8657, Japan

2 Tokyo Pest Control Association, 2-9-8 Kajicho, Chiyoda-ku, Tokyo 101-0044, Japan

3 Department of Biological Sciences, Fordham University, 441 East Fordham Road, Bronx, NY 10458, USA
- The predominance of roof rats in Tokyo may explain the limited effects on business activities

\section{Introduction}

Brown rats (Rattus norvegicus) and roof rats (Rattus rattus) have been regarded as pests for thousands of years around the world. In addition to economic damage, rats are known to cause many zoonoses, including bubonic plague (Barnett 1948) and leptospirosis (Seijo et al. 2002). Even today, $17 \%$ and $5 \%-35 \%$ of brown rats have been reported to carry zoonotic leptospires (Koizumi et al. 2009) and helminths (Banzai et al. 2018), respectively, in Tokyo, Japan, a metropolis with the largest number of residents globally (Cox 2021). Given that most zoonoses associated with rats are transmitted by direct contact with rats (e.g., rat bite fever) or their excretions (e.g., leptospirosis and hemorrhagic fever with renal syndrome), ingestion of water and foods contaminated by excretions (e.g., salmonellosis), or the ectoparasites 
of rats (e.g., bubonic plague), the presence of rats in buildings poses serious risks to human health.

Because rats are commensal organisms that depend on human activities for food, rats are thought to modify their behavior very quickly when humans alter their activities in response to an event. Based on this assumption, the importance of rat control as a means of preventing infestations in buildings and the spread of zoonoses is typically announced following natural disasters, especially in North America, because disasters can alter human activities in the affected areas. However, surprisingly, little data are available on the immediate effects of changes in human activities on rat populations. While several studies have analyzed the effects of disasters on rats (Htwe et al. 2013; Peterson et al. 2020), these studies were performed several years after the disasters occurred. As a result, they provided information on how rats adapted to newly established human activities after the disasters occurred, rather than on how rats responded to sudden shifts in human activity. In urban areas, the habitats of brown rats and roof rats are usually segregated (Cavia et al. 2009; Worth 1950). Consequently, these two rat species are considered to rely on different types of human activity for obtaining food. In addition, the balance between brown rats and roof rats in urban centers appears to differ across countries. For example, although no data are currently available, it is widely accepted that brown rats are predominant in North America (Battersby et al. 2008). However, anecdotal evidence obtained from Japanese pest management professionals suggests that roof rats are predominant in Tokyo (Harunari et al. 2009; Yabe 1997a, 1997b; Yabe et al. 2000). It is therefore possible that shifts in human activities differentially affect these two rat species and are associated with the differing outcomes in rat populations among countries. In the spring of 2020, the novel coronavirus SARS-CoV-2 caused the COVID-19 pandemic. Social distancing measures were implemented globally to curtail the spread of SARSCoV-2, which significantly altered human activities. Thus, the pandemic presented a unique opportunity to obtain information regarding the immediate effects of shifts in human activities on rat populations in a variety of countries. Following the implementation of social distancing measures, increased sightings of rats in urban slums were reported in Brazil (Neves Souza et al. 2021). Increased sightings of rats in public spaces in the USA and UK were also frequently broadcasted in English language media. However, it is unclear whether these rats invaded and infested buildings. In addition, it was unclear how social distancing measures affected rats in the other countries.

The present study was conducted to clarify these points. We first analyzed the changes in the number of public service calls in Tokyo to assess whether rat sightings increased after the implementation of social distancing measures. We then surveyed the changes in business activities of pest management professionals in the USA, Canada, and Tokyo. The respondents in Tokyo were additionally asked to estimate the proportions of brown rats, roof rats, and house mice that they had seen or captured before and after implementation of the social distancing measures. Residents in these countries would hire pest management professionals when the degree of rat infestations in their buildings became unacceptable. In addition, it was demonstrated that pest management professionals could be a valuable source of information regarding urban pests (Himsworth et al. 2013). Therefore, the business activities of pest management professionals can be used to infer the extent to which social distancing measures resulted in rat infestations in these countries.

\section{Materials and methods}

\section{Social distancing measures in the USA, Canada, and Tokyo.}

In the USA, a state of emergency was first declared in Washington State on February 29, 2020. The measure was extended to include all 50 states when the state of Iowa declared a state of emergency on March 17. Among the 10 provinces of Canada, seven and two provinces declared a state of emergency and a public health emergency, respectively, from March 13 (Québec Province) to March 22 (Nova Scotia Province). The last province to implement such a measure was Prince Edward Island Province on April 16. In Japan, the Government declared a state of emergency in Tokyo on April 7, 2020. The declaration was expanded to include the entire country on April 16.

In order to analyze the immediate effects of shifts in human activities, we examined the changes that occurred over an approximately one-month period after the declaration of the state of emergency.

\section{Analysis of the number of public service calls in Tokyo}

The data for the number of public service calls in Tokyo were kindly provided by the Tokyo Pest Control Association (TPCA). The TPCA is a public interest incorporated association with 105 pest management corporate members. Residents in Tokyo can call the TPCA when they find rodents in and/or close to their residential areas. Other than the cost of the local call, calls to the TPCA and subsequent consultations are free of charge. The TPCA will introduce its members when necessary, which allows people to decide whether or not they will call and enter into a contract with the introduced companies. In Tokyo prefecture, which comprises 23 wards, 26 cities, 3 towns, and 1 village, the distribution of accommodation, eating, and drinking services 
(79.9\% in 2016, based on the Tokyo Metropolitan Government website) and people $(69.2 \%$ in 2020 , based on the Tokyo Metropolitan Government website) are more heavily concentrated in the eastern 23 wards. We therefore focused on the calls made to the TPCA in the 23 wards. The total number of calls in March and April from 2017 through 2020 was analyzed. The number of calls that differed by more than 2 standard deviations from the average for the same month in the previous three years was detected using Microsoft Excel. Given that these samples represent $4.4 \%$ of the population $(P=0.044)$, these numbers were classified as "increased" or "decreased" according to the changes from the average. However, because the classification seemed to be relatively unreliable, the change was regarded as "unchanged" when both the number in the target month and the average for the same month in the previous three years were less than five. In order to specify the location, we further analyzed the changes in the number of calls in each ward in the same way. The differences in the number of wards classified as "increased," "decreased," or "unchanged" in March and April in 2019 and 2020 were analyzed by Fisher's exact test using R (R Core Team 2021). The significance level was set at $P<0.05$.

\section{Analysis of the survey among pest management professionals}

The survey among pest management professionals was conducted with the approval of the Ethics Review Committee of the Faculty of Agriculture at The University of Tokyo. On May 5, 2020, a Google Forms-based English questionnaire was distributed to the pest management professionals in the USA via Pest Control Magazine and in Canada through the Canadian Pest Management Association. The Canadian Pest Management Association is a national conglomerate that coordinates the activities of all provincial and regional pest control associations in Canada. We also posted the English questionnaire on social media outlets, such as Twitter (April 30) and LinkedIn (April 22), to solicit pest management professionals to participate in the survey. The responses and timestamps were recorded on a server when the respondents pressed the "submit" button at the end of the questionnaire. In Tokyo, the questionnaire was translated into Japanese and five sheets were sent to the headquarters of each 105 TPCA member by post on May 11, 2020. It is known that almost all the professionals in Tokyo work in multiple wards. In addition, we wanted to emphasize that we are collecting answers from each pest management professional, rather than one representative answer from each company. Furthermore, we wanted to increase the number of responses, rather than to analyze a response rate. Therefore, the following notes were clearly indicated in the attached cover letter: "Multiple responses from one company are accepted"; "Responses can differ among respondents from the same company"; "One respondent can complete questionnaires for multiple cities"; "Please use one questionnaire sheet for each subject city"; "Please make photocopies of the questionnaire sheet when necessary." The respondents were asked to send their responses to the Laboratory of Veterinary Ethology, The University of Tokyo by fax or by post.

Because we did not collect personally identifiable information, we first requested the online respondents to agree to the following points and press the "Agree" button: "You are currently working as a pest management professional"; "You have read the entire consent form"; "You are voluntarily participating in this survey." In the survey performed in Tokyo, these requirements were clearly indicated at the top of the questionnaire sheet. The respondents were then requested to provide the name of the subject city and to answer the following three questions: 1) "What have been the effects of social distancing measures on the overall amount of ratrelated business in the subject city?", 2) "What have been the effects of social distancing measures on the amount of rat-related business from new clients in the subject city?", and 3) "What is the approximate proportion of rat-related business from new clients in the subject city?" The respondents were required to select one of four possible answers for questions 1 and 2: "Unchanged," "Increased," "Decreased," and "Prefer not to answer," and one of six possible answers for question 3: "None," "1-25\%," "26-50\%," "51-75\%," "76-99\%," "All are from new clients," and "Prefer not to answer." The differences in the responses to each question among three countries were analyzed by Fisher's exact test using R (R Core Team 2021). Based on Bonferroni's correction, the significance level was set at $P<0.017$. The triple $P$ value was reported as the adjusted $P$ value for conciseness.

Furthermore, as question 4, the respondents in Tokyo were additionally asked to estimate the proportions of brown rats, roof rats, and house mice that they had seen or captured in the subject city before and after the implementation of social distancing measures. The effects of social distancing measures were analyzed by Fisher's exact test using $\mathrm{R}$ ( $\mathrm{R}$ Core Team 2021). The significance level was set at $P<0.05$. Details of additional analyses in Japan are provided in the Supplementary Information.

\section{Results and discussion}

In 2020, although the total number of calls in March $(n=83)$ was unchanged, the number of calls in April $(n=87)$ had increased compared to the same month in the previous three years. To confirm whether the change was specific to 2020 , we analyzed the number of calls in previous years. In 2019, the total number of calls in March $(n=61)$ and April $(n=71)$ had decreased and was unchanged, respectively. We found 
no changes in the number of calls in March (2018: $n=76$, 2017: $n=81)$ and in April (2018: $n=69,2017: n=70)$ in 2018 and 2017. These results suggest that social distancing measures increased the total number of calls in April 2020. To clarify the pattern of changes, we further analyzed the changes in each ward. In April 2020, the number of calls was found to have increased in wards with a large downtown area, including Shibuya and Ueno (Fig. 1). However, when we compared the number of wards where the changes occurred, the differences in the numbers in March and April were not statistically significant between 2019 and 2020 (March: $P=0.053$, April: $P=0.19$ ) (Figs. 1 and S1). These

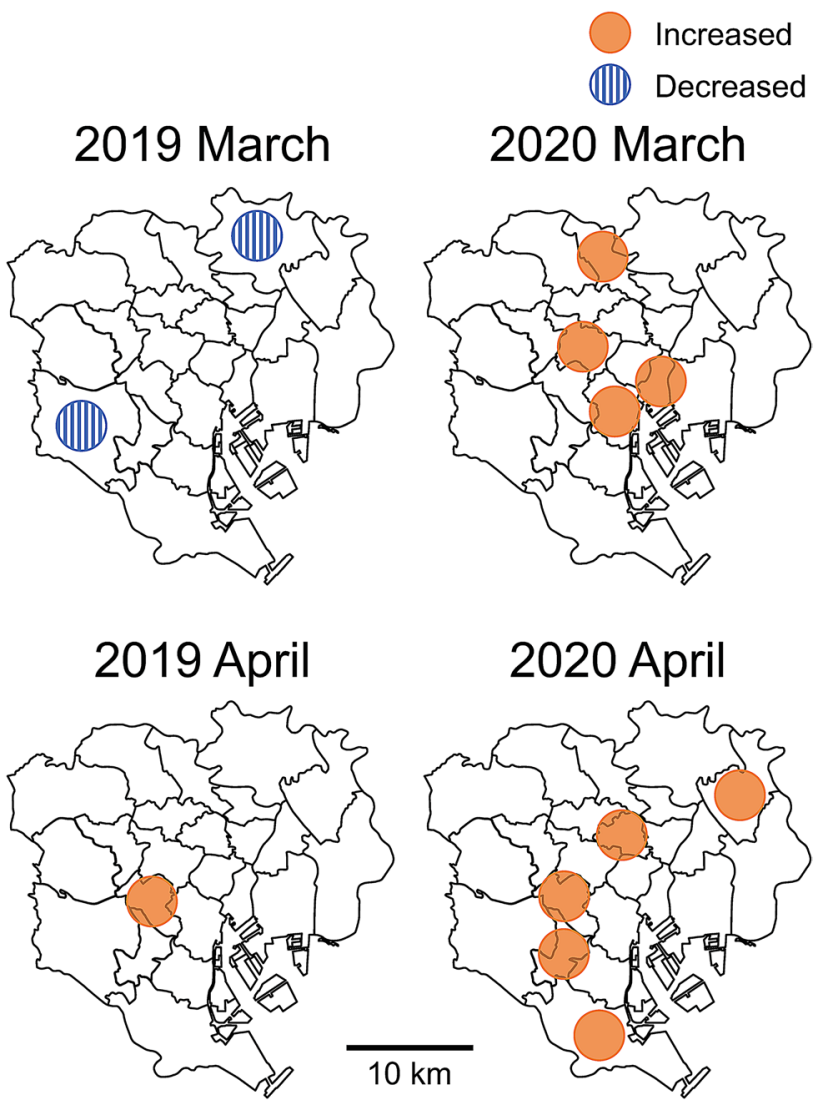

Fig. 1 Schematic diagrams showing the locations where the number of calls to Tokyo Pest Control Association increased (solid orange circle) or decreased (striped blue circle) compared to the previous three years in March and April of 2019 and 2020

results suggest that the changes occurred in a limited number of wards, rather than in a large number of wards. Taken together, these results suggest that social distancing measures resulted in increased rat sightings in Tokyo.

In the survey, we obtained 32,23 , and 77 responses from the pest management professionals working in the USA, Canada, and Tokyo, respectively. These responses represented 29 cities in 18 states of the USA, 20 cities in four provinces of Canada, and all 23 wards of Tokyo. The respondents in North America who selected "Prefer not to answer" were excluded from the analysis (ranging from $n=1$ to $n=4$ ). Fisher's exact test revealed that the responses to all questions differed among the three countries (adjusted $P s<0.01$ ), except for the responses to question 1 between the USA and Canada (adjusted $P=0.53$ ) (Table 1). In question 1, we asked about the changes in the overall amount of business. The most common response in the USA (51.6\%) and Canada (60.9\%) was "Increased" (Fig. 2a). In contrast, "Unchanged" was the most common response in Tokyo $(62.3 \%)$. In question 2, we asked about the changes in the amount of business from new clients. The most common response was "Increased" in the USA (53.6\%) and Canada (54.5\%), whereas "Unchanged" was the most common response in Tokyo (71.4\%) (Fig. 2b). In question 3, we asked about the proportion of the amount of business from new clients. Although " $1-25 \%$ " was the most common response in the three countries (USA: $26.7 \%$, Canada: $31.8 \%$, Tokyo: $59.7 \%)$, "all are from new clients" was chosen only in the USA (6.7\%) and Canada (18.2\%) (Fig. 2c).

In Tokyo, it is possible that the proportions of brown rats and roof rats changed following the implementation of social distancing measures, even if the business activities of pest management professionals were less affected. To assess this possibility, the fourth question asked Japanese pest management professionals what proportion of different rodent species were caught and/or sighted in Tokyo. However, Fisher's exact test revealed that social distancing measures did not affect the relative proportions of rodent species $(P=1)$ (Table 1). Both before and after social distancing measures were implemented, roof rats were considered to be the predominant rodents in Tokyo (Table 2). It is also possible that the 23 wards of Tokyo are a unique area in Japan in that the effects of social distancing measures were extremely limited. To assess this possibility, we analyzed 35 responses to the same questionnaire from cities outside the 23 wards of Tokyo; this sample comprised 20 cities in 15 prefectures (Supplementary Information). Fisher's exact test revealed that the responses to all questions differed between Tokyo and these 20 other cities (question 1: $P<0.01$, question 2: $P<0.05$, question 3: $P<0.01$ ) (Table S1). For question 1, the proportion of "Unchanged" was $82.9 \%$ in other cities and $62.3 \%$ in Tokyo (Fig. S2a). Similarly, for question 2, the proportion of "Unchanged" was $82.9 \%$ in other cities and $71.4 \%$ in Tokyo (Fig. S2b). For question 3, the most common response was "None" in other cities (57.1\%), whereas "1-25\%" was the most common response in Tokyo (59.7\%) (Fig. S2c). Therefore, Tokyo seemed to be the most affected area, rather than the least affected area in Japan.

Taken together, the findings of the present survey suggested that the business activities of pest management professionals in North America were increased following the implementation of social distancing measures. In addition, 
the measures differentially affected the amount of business from new clients in the USA and in Canada. Conversely, limited effects attributed to social distancing measures were observed among pest management professionals in Tokyo.

Based on the findings in the present study, we suggest that the immediate effects of social distancing measures differed between North America and Tokyo. In North America, English language media outlets stated that social distancing measures were associated with an increase in rat sightings. The findings of the present study implied that rat infestations were increased with the implementation of social distancing measures. In contrast, few infestations appeared to be attributed to social distancing measures in Tokyo, even if rat sightings increased. However, we should also acknowledge several limitations in this study. For example, the number of responses in the survey was too small to adequately capture the entire picture in each country. It is also possible that numerous responses were answered by a small number of the same professionals, although this seems unlikely based on the timestamps of the responses. Furthermore, the results may have changed when the pandemic and subsequent implementation of social distancing measures occurred in different seasons. Future analyses based on a larger number of responses from both the northern and southern hemispheres would be helpful to clarify the immediate effects of social distancing measures on rat populations.

One possible interpretation of the results for Tokyo could be that social distancing measures did not affect the rats in Tokyo. For example, residents have started to stay at home at different times, and this could lead to new sightings in residential areas even if the rat population itself has not changed at all. However, it is more likely that the relatively fewer effects in Tokyo may have been due to the predominance of roof rats in Tokyo. Specifically, it is possible that that social distancing measures differentially affected brown rats and roof rats. In Tokyo, brown rats are found outside of buildings in downtown areas where they forage on the garbage that is disposed of by restaurants and bars and placed curb-side at midnight for collection the following morning (Yabe 2021). Social distancing measures might have forced brown rats to forage in new areas in order to find alternative food sources because restaurants and bars closed early at night and the quantities of garbage placed curb-side decreased dramatically. Because brown rats do not travel long distances in urban areas (Combs et al. 2018; Gardner-Santana et al. 2009), rat sightings would only have increased in a limited number of wards. However, the increased sightings of brown rats did not result in rat infestations in buildings that were serious enough to prompt residents to hire pest management professionals. One of the reasons may be that the number of roaming brown rats was estimated to be small. The present study suggested that the proportion of brown rats in Tokyo was approximately $20 \%$. In addition, compared to roof rats, brown rats required a larger diameter of hole to pass through (Pitt et al. 2011). Therefore, it was difficult for brown rats to invade buildings in Tokyo because buildings are constructed to prevent entry by roof rats. Unlike brown rats, roof rats inhabit building interiors and feed on food stocks, grease vats, and used oil in the kitchens of restaurants and bars (Tanikawa et al. 2007). Given that restaurants and bars remained open for takeaway meals in the daytime, the food sources for roof rats might have been less affected by social distancing measures. In addition,
Table 1 Results of statistical analyses

\begin{tabular}{lccc}
\hline & $\mathrm{df}$ & $\chi^{2}$ value & $P$ value \\
\hline Question 1: Changes in the overall amount of business & & & \\
USA (31) vs. Canada (23) & 2 & 3.50 & 0.178 \\
USA (31) vs. Tokyo (77) & 2 & 44.8 & $4.17 \times 10^{-11}$ \\
Canada (23) vs. Tokyo (77) & 2 & 65.1 & $2.09 \times 10^{-16}$ \\
Question 2: Changes in the amount of business from new clients & & & \\
USA (28) vs. Canada (22) & 2 & 21.5 & $2.81 \times 10^{-6}$ \\
USA (28) vs. Tokyo (77) & 2 & 56.4 & $3.49 \times 10^{-14}$ \\
Canada (22) vs. Tokyo (77) & 2 & 67.2 & $7.09 \times 10^{-18}$ \\
Question 3: Proportion of the amount of business from new clients & & & \\
USA (30) vs. Canada (22) & 5 & 15.7 & 0.00694 \\
USA (30) vs. Tokyo (77) & 5 & 29.3 & $8.90 \times 10^{-6}$ \\
Canada (22) vs. Tokyo (77) & 5 & 41.2 & $6.60 \times 10^{-9}$ \\
Question 4: Proportions of rodent species in Tokyo & & & \\
Before (77) vs. After (77) & 2 & 0.030 & 1 \\
\hline
\end{tabular}

The numbers of samples are shown in parentheses

Data were analyzed by Fisher's exact test

df: degree of freedom 


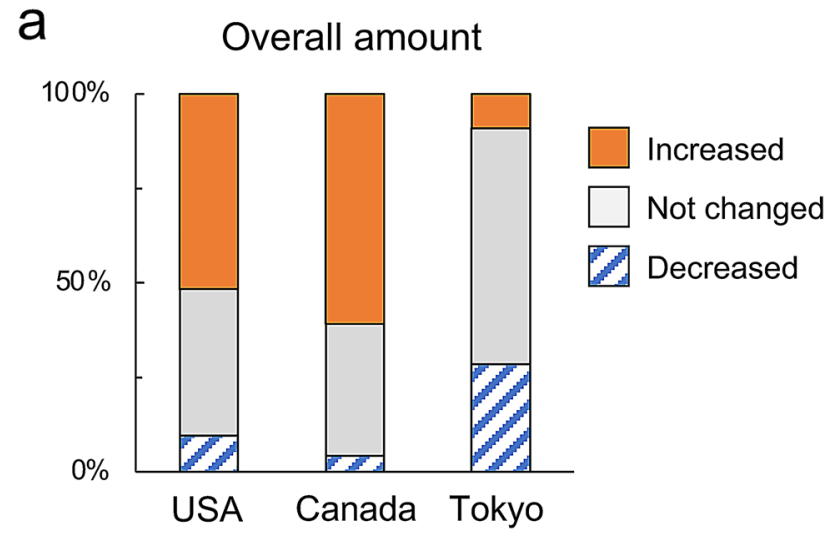

b Amount from new clients

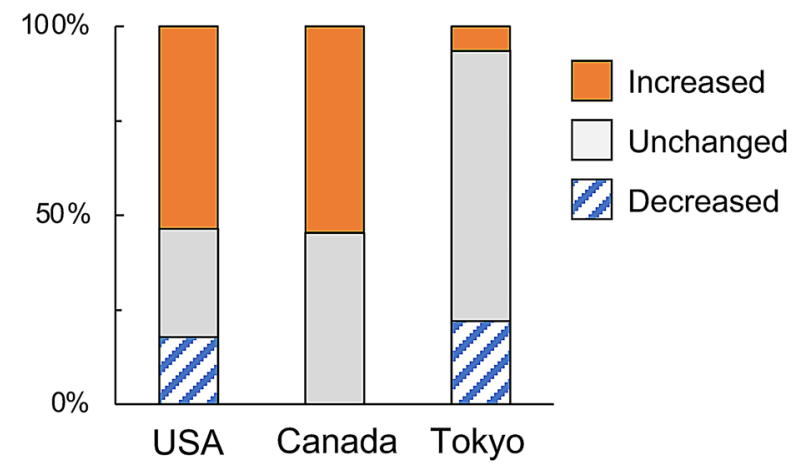

C Proportion of new clients

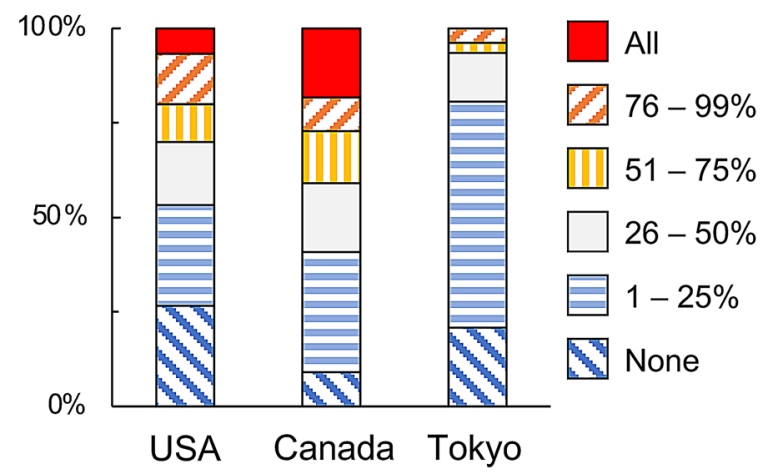

Fig. 2 Responses from pest management professionals in the USA, Canada, and Tokyo. The effects of social distancing measures on (a) overall amount of rat-related business in the subject city, (b) the amount of rat-related business from new clients in the subject city, and (c) the approximate proportion of the amount of rat-related business from new clients in the subject city during the month after the implementation even if the availability of food is reduced, roof rats appear to be reticent to forage widely and compete with humans for food. For example, in a building with many restaurants on the 6th floor in Tokyo, roof rats were trapped continuously on the same floor for a further 46 days after the closure of the building (Tanikawa and Uchida 2000). The findings of that study were likely not attributed to the presence of a small number of outliers, because more than $4 \%$ of the trapped rats were trapped one month after the closure of the building (Tanikawa and Uchida 2000). Therefore, it is assumed that most populations of roof rats remained in their habitats when social distancing measures were implemented. Taken together, it appears that very few rat infestation problems were attributed to the implementation of social distancing measures in Tokyo. This scenario is consistent with one of the authors' personal experience; in decades of being engaged in pest control operations in collaboration with the Japanese Government, he (TT) has not observed any increase in rat infestations in urban areas after a natural disaster in Japan.

In summary, we found that social distancing measures differentially affected rat populations in North America and Tokyo. In North America, the business activities of pest management professionals increased in conjunction with the increase in the reports of rat sightings in the media. In contrast, social distancing measures in Tokyo had very little impact on the activities of pest management professionals, even though rat sightings increased. Based on differences in the predominant rodent species between North America and Tokyo, we hypothesized that the magnitude of rat infestations associated with shifts in human activities decreases when the relative proportion of roof rats increases. After natural disasters that change human activities, a certain amount of resources should be allocated to rat control in order to maintain the public health of affected residents. On the other hand, since resources are limited immediately after disasters, channeling resources toward rat control can have an adverse effect on other restoration activities. It is therefore crucial to extrapolate the extent of possible rat infestations in disaster-affected areas as accurately as possible. Further research focusing on the relationship between the proportion of rodent species and the degree of rat infestations could facilitate optimal resource balancing between remediation efforts related to the disaster and protecting the public health of affected inhabitants. 
Table 2 Proportions of rodent species in Tokyo before and after the implementation of social distancing measures

\begin{tabular}{lcl}
\hline & $\begin{array}{l}\text { Before the } \\
\text { implementation }(\%)\end{array}$ & $\begin{array}{l}\text { After the implementation } \\
(\%)\end{array}$ \\
\hline Brown rats & $20.5 \pm 1.7$ & $21.5 \pm 2.0$ \\
Roof rats & $78.7 \pm 1.8$ & $77.7 \pm 2.0$ \\
House mice & $0.9 \pm 0.4$ & $0.9 \pm 0.3$ \\
\hline
\end{tabular}

Data are expressed as the mean \pm standard error of the mean

Supplementary Information The online version contains supplementary material available at https://doi.org/10.1007/s10340-021-01405-z.

Acknowledgements This study was supported by JSPS KAKENHI (Grant Numbers 20H03160 and 20H04766). We thank Pest Control Magazine, the National Pest Management Association of North America, and the Japan Pest Control Association for their support and participation.

Authors' contributions $\mathrm{YK}$ and MP conceived and designed the research; YK, TT, and MP collected the data; YK and MO analyzed the data; YK and MP wrote the manuscript. All authors read and approved the manuscript.

Funding This study was supported by JSPS KAKENHI (Grant Numbers $20 \mathrm{H} 03160$ and 20H04766).

Data Availability The datasets generated during and/or analyzed during the current study are available from the corresponding author on reasonable request.

\section{Declarations}

Conflicts of interest The authors declare that they have no conflicts of interest.

Consent to participate We did not collect personally identifiable information in the survey. Nonetheless, informed consent was obtained from all individual participants included in the study.

\section{References}

Banzai A, Tanikawa T, Kimura G, Sakai T, Kawakami Y (2018) Parasitic helminths collected from the brown rat, Rattus norvegicus, in Chuo ward, Tokyo, Japan. Med Entomol Zool 69:171-176. https:// doi.org/10.7601/mez.69.171

Barnett SA (1948) Rat control in a plague outbreak in Malta. J Hyg (lond) 46:10-18. https://doi.org/10.1017/s0022172400036019

Battersby S, Hirschhorn RB, Amman BR (2008) Commensal rodents. In: Bonnefoy X, Kampen H, Sweeney K (eds) Public health significance of urban pests. World Health Organization Regional Office for Europe, Copenhagen, pp 387-419

Cavia R, Cueto GR, Suarez OV (2009) Changes in rodent communities according to the landscape structure in an urban ecosystem. Landsc Urban Plan 90:11-19. https://doi.org/10.1016/j.landu rbplan.2008.10.017
Combs M, Byers KA, Ghersi BM, Blum MJ, Caccone A, Costa F, Himsworth CG, Richardson JL, Munshi-South J (2018) Urban rat races: spatial population genomics of brown rats (Rattus norvegicus) compared across multiple cities. Proc Biol Sci 285:285. https://doi.org/10.1098/rspb.2018.0245

Cox W (2021) Demographia world urban areas, 17th edn., Wendell Cox Consultancy, http://www.demographia.com Accessed 20 June 2021.

Gardner-Santana LC, Norris DE, Fornadel CM, Hinson ER, Klein SL, Glass GE (2009) Commensal ecology, urban landscapes, and their influence on the genetic characteristics of city-dwelling Norway rats (Rattus norvegicus). Mol Ecol 18:2766-2778. https://doi.org/ 10.1111/j.1365-294X.2009.04232.x

Harunari T, Tanikawa T, Nihei N, Komagata O, Kobayashi M (2009) A trial to employ the city geographical information system to determine the population of rats in a residential area. Pestology 24:47-50

Himsworth CG, Feng AYT, Parsons K, Kerr T, Patrick DM (2013) Using experiential knowledge to understand urban rat ecology: a survey of Canadian pest control professionals. Urban Ecosyst 16:341-350. https://doi.org/10.1007/s11252-012-0261-4

Htwe NM, Singleton GR, Nelson AD (2013) Can rodent outbreaks be driven by major climatic events? evidence from cyclone Nargis in the Ayeyawady Delta, Myanmar. Pest Manag Sci 69:378-385. https://doi.org/10.1002/ps.3292

Koizumi N, Muto M, Tanikawa T, Mizutani H, Sohmura Y, Hayashi E, Akao N, Hoshino M, Kawabata H, Watanabe H (2009) Human leptospirosis cases and the prevalence of rats harbouring Leptospira interrogans in urban areas of Tokyo, Japan. J Med Microbiol 58:1227-1230. https://doi.org/10.1099/jmm.0.011528-0

Neves Souza F, Awoniyi AM, Palma FAG, Begon M, Costa F (2021) Increased rat sightings in urban slums during the COVID-19 pandemic and the risk for rat-borne zoonoses. Vector Borne Zoonotic Dis 21:160-161. https://doi.org/10.1089/vbz.2020.2686

Peterson AC, Ghersi BM, Campanella R, Riegel C, Lewis JA, Blum MJ (2020) Rodent assemblage structure reflects socioecological mosaics of counterurbanization across post-Hurricane Katrina New Orleans. Landsc Urban Plan 195:103710. https://doi.org/ 10.1016/j.landurbplan.2019.103710

Pitt WC, Sugihara RT, Driscoll LC, Vice DS (2011) Physical and behavioural abilities of commensal rodents related to the design of selective rodenticide bait stations. Int J Pest Manag 57:189-193. https://doi.org/10.1080/09670874.2011.561889

R Core Team (2021) R: a language and environment for statistical computing. R Foundation for Statistical Computing, Vienna, Austria. https://www.R-project.org/.

Seijo A, Coto H, San Juan J, Videla J, Deodato B, Cernigoi B, Messina OG, Collia O, de Bassadoni D, Schtirbu R, Olenchuk A, de Mazzonelli GD, Parma A (2002) Lethal leptospiral pulmonary hemorrhage: an emerging disease in Buenos Aires, Argentina. Emerg Infect Dis 8:1004-1005. https://doi.org/10.3201/eid0809.010499

Tanikawa T, Uchida A (2000) Changes of trapped numbers and age composition of the roof rat, Rattus rattus, by glue traps in an urban building. Pest Cont Res 15:1-4

Tanikawa T, Ikejiri Y, Xie L (2007) Population estimating method of roof rats, Rattus rattus, by the continuous capture. House Househ Insect Pest 28:133-137

Worth B (1950) Field and laboratory observations on roof rats, Rattus rattus (Linnaeus), in Florida. J Mammal 31:293-304. https://doi. org/10.2307/1375298

Yabe T (1997a) Changes in species composition of the Norway rat (Rattus norvegicus) and the roof rat (R. rattus) in urban area. Med Entomol Zool 48:285-294. https://doi.org/10.7601/mez.48.285 
Yabe T (1997b) Probable relation of increase in big buildings and road coverage to the rise and decline of Rattus rattus in Sapporo, Japan. Med Entomol Zool 48:251-255. https://doi.org/10.7601/mez.48. 251

Yabe T (2021) Prologue: Commensal rodent problems across the globe. In: Mulungu LS (ed) Rodents. IntechOpen. https://doi.org/10. 5772/intechopen.95828
Yabe T, Asai R, Takagi Y, Kohsaki KI (2000) Reason for the recent increase of roof rat infestation in residential areas in Tokyo. Med Entomol Zool 51:211-213. https://doi.org/10.7601/mez.51.211

Publisher's Note Springer Nature remains neutral with regard to jurisdictional claims in published maps and institutional affiliations. 